\title{
ON THE UV CET-TYPE STAR ROTATION
}

\author{
R. E. GERSHBERG \\ Crimean Astrophysical Observatory, U.S.S.R.
}

(Paper read by R. P. Kraft)

\begin{abstract}
The upper limits of flare star rotational velocities are evaluated from the photometric features of the stars and on the basis of the widths of the $\mathrm{H} \alpha$ emission line in the quiet chromospheres. The rotational velocity of UV Cet does not exceed $22 \mathrm{~km} / \mathrm{sec}, Y Z$ CMi: $37 \mathrm{~km} / \mathrm{sec}$, the value $v \sin i$ of AD Leo does not exceed $52 \mathrm{~km} / \mathrm{sec}$, and that of EV Lac: $25 \mathrm{~km} / \mathrm{sec}$.
\end{abstract}

\section{Introduction}

The observational data and some theoretical considerations suggest that the UV Cet-type flare stars should have an appreciable rotation. Krzeminski [1] has found periodic brightness variations of $\mathrm{dMe}$-stars while the $\mathrm{dM}$-stars investigated did not reveal such features. Krzeminski concluded that the most probable interpretation of such events is a rotation effect of a spotted star. This hypothesis was discussed earlier by Chugainov [2] in connection with similar photometric features of the emission dwarf HDE 234677. Then, the flare quasi-periodicity of UV Cet-type stars discovered by Andrews [3] and by Chugainov [4] can also be explained by the rotation effect of a star with an active region (or regions) where flares occur. Finally, spectroscopic and radio investigations of UV Cet-type star flares give more and more evidence of the similarity between those stellar processes and solar chromospheric flares [5] i.e. they give new arguments in favor of the electromagnetic nature of the UV Cet-type star flare activity which is probably connected with stellar rotation in the end [6].

Rotational velocity determinations for flare stars have not been carried out to date. In this paper probable rotational velocities of UV Cet, YZ CMi, AD Leo and EV $\mathrm{Lac}$ - the stars being under detailed photometric investigation - are discussed.

\section{UV Cet}

UV Cet spectrograms obtained to date do not permit evaluation of the rotational velocity of the star by the classical method. Therefore nothing else is left but the method of indirect evaluations. The UV Cet rotational velocity can be evaluated on the basis of photometric features of this star.

In the wide spectral regions of the photometric UBV system, mean deviations in the dM-star energy distribution from the Planck function are small; therefore the spectrophotometric temperature of radiation which can be determined from the $B-V$ color may be supposed to be close enough to the photovisual brightness temperature of the star. Then, the color $B-V=+1^{\mathrm{m}} .9$ gives $T(\mathrm{UV}$ Cet $)=2700 \mathrm{~K}$. This value is smaller than the effective temperature for a dM 5.5 star in Johnson's scale [7]: $T_{\text {eff }}(\mathrm{dM} 5.5) \approx 3000 \mathrm{~K}$, but according to Tsuji [8], this scale is overstated by $200 \mathrm{~K}$ for M-stars. 
Spectral classes of the L-726-8 system components are very close, therefore the UV Cet part of the total luminosity of the system can be found as follows:

$$
L_{\mathrm{B}}(\mathrm{UV} \text { Cet })=\frac{10^{-0.4 \Delta m}}{1+10^{-0.4 \Delta m}} \cdot L_{B}(\mathrm{~L}-726-8 \mathrm{AB})
$$

and $\Delta m=0.5$ for this system. Finally, the photometric radius of UV Cet may be calculated from the expression

$$
R(\text { UV Cet })=\sqrt{\frac{L_{B}(\mathrm{~L}-726-8 \mathrm{AB}) f(\Delta m)}{4 \pi \int B_{\lambda}(2700 \mathrm{~K}) \varphi_{B}(\lambda) \mathrm{d} \lambda}}=5.6 \times 10^{9} \mathrm{~cm}\left(0.08 R_{\odot}\right)
$$

where

$$
f(\Delta m)=\frac{10^{-0.4 \Delta m}}{1+10^{-0.4 \Delta m}},
$$

$B_{\lambda}(T)$ is the Planck function and $\varphi_{B}(\lambda)$ is the response curve of the B-band.

According to Chugainov [5], the characteristic time interval between the flares of UV Cet is 4.4 . If one identifies this time interval with a stellar rotational period, the equatorial velocity of rotation will be equal to $22 \mathrm{~km} / \mathrm{sec}$; if the real brightness temperature is within the range $2700 \pm 200 \mathrm{~K}$, the rotational velocity will be from 15 to $35 \mathrm{~km} / \mathrm{sec}$. But the main uncertainty of that evaluation is due to identifying the characteristic time interval between flares with the rotational period. On the one hand, the $4^{\mathrm{h}} .4$ period may be a multiple of the real one, and Australian astronomers were inclined to draw such a conclusion when they found two strong flares of UV Cet separated by a time interval of less than 2 hours [9]; in that case the real rotational velocity may be a few times higher. On the other hand there may be several active regions on the stellar surface and then the real rotational velocity may be several times lower than the one evaluated above. The second case seems to be more likely; therefore the value $22 \mathrm{~km} / \mathrm{sec}$ must be regarded as an upper limit of the real rotational velocity.

\section{YZ CMi}

The photometric radius of YZ CMi is equal to $1.4 \times 10^{10} \mathrm{~cm}\left(0.21 R_{\odot}\right)$. The characteristic time interval between flares is $6^{\mathrm{h}} 50^{\mathrm{m}}$ [4]. Therefore a 'photometric' rotational velocity is $37 \mathrm{~km} / \mathrm{sec}$. But YZ CMi is one of several flare stars whose duplicity is not known. The magnitude $f(\Delta m)$ is then taken to be unity. If YZ CMi is an inseparable binary the photometric radius and 'photometric' rotational velocity must be multiplied by the function $[f(\Delta m)]^{1 / 2}$ which is equal to $0.71-0.24$ for $\Delta m=0^{\mathrm{m}}-3^{\mathrm{m}}$.

In order to obtain an independent upper limit of $v \sin i$ for YZ CMi, 4 spectrograms in the red region of the spectrum were taken with the Shajn $2.6 \mathrm{~m}$ reflector; the short focus camera $(24 \AA / \mathrm{mm})$ and high sensitive A-700 emulsion were used. The $\mathrm{H} \alpha$ stellar emission line and 2-4 neon lines were measured photometrically on each film; neon lines were chosen close to $\mathrm{H} \alpha$ in terms of wavelengths and intensities. In 
principle, an observed width of $\mathrm{H} \alpha$ at half-intensity level is determined by an instrumental profile, by Doppler and Stark effects and by self-absorption. Because hydrogen and neon line profiles have no appreciable wings on our spectrograms, the real half widths were calculated as a square root of an observed $\mathrm{H} \alpha$ half width squared minus a neon half width squared. Half widths of $\mathrm{H} \alpha$ in $\mathrm{YZ} \mathrm{CMi} \mathrm{spectrograms} \mathrm{reduced} \mathrm{in}$ this way are equal to 1.0-1.4 $\AA$. Neither thermal motion nor Stark effect can supply such wide lines under chromospheric conditions. We have no information on the character of macroscopic motion in the chromosphere of YZ CMi nor on its optical thickness at the $\mathrm{H} \alpha$ line; therefore one can take the entire reduced half width to be due to rotation effects and one is able to evaluate $(v \sin i)_{\max }$ as less than $46 \mathrm{~km} / \mathrm{sec}$.

\section{AD Leo}

The photometric radius of $\mathrm{AD}$ Leo is equal to $2.8 \times 10^{10} \mathrm{~cm}$. But $\mathrm{AD}$ Leo is a known binary $[10,11]$. According to [10], the satellite mass is $0.03 M_{\odot}$ and it is natural to jdentify this satellite as a flare star of the AD Leo system. Supposing $\Delta m=2^{\mathrm{m}}-3^{\mathrm{m}}$, one can find $R=0.15-0.10 R_{\odot}$. Thus the flare star of the AD Leo system is probably very similar to UV Cet. But the available flare statistics do not give a characteristic time interval between AD Leo flares.

An upper limit of $v \sin i$ for AD Leo was evaluated on the basis of spectral data: $(v \sin i)_{\max }<52 \mathrm{~km} / \mathrm{sec}$. According to Herbig's communication, widths of absorption spectrum lines give $(v \sin i)_{\max }<15 \mathrm{~km} / \mathrm{sec}$ for AD Leo, but the absorption spectrum of this system is determined by the main component and the magnitude mentioned may bear no relation to the flare star.

\section{EV Lac}

The photometric radius of EV Lac is equal to $0.22 R_{\odot}$, but EV Lac is a probable binary [12] and that figure may be an upper limit to a flare star radius. The search for a characteristic time interval between EV Lac flares has not been undertaken to date, but the latest observations of this star give a new argument in favor of the spotted star hypothesis [13]: slow and considerable brightness variations were found during monitoring of the star in the UV region and stellar flares were occurring only in brightness minima; one may think again that there is an active region (or regions) with a lower temperature - stellar spots - where flares occur. A similar photometric feature was found during the latest UV Cet brightness monitoring in the ultraviolet [14].

In 1960 Wilson [15] obtained an EV Lac spectogram with dispersion $9 \AA / \mathrm{mm}$ with the Palomar reflector. He has estimated $v \sin i<25 \mathrm{~km} / \mathrm{sec}$ from the hydrogen emission lines. The Crimean spectrogram taken with dispersion $24 \AA / \mathrm{mm}$ gives a higher limit: $v \sin i<54 \mathrm{~km} / \mathrm{sec}$. However this result must not lead to the suspicion that all our spectrographic evaluations of $v \sin \mathrm{i}$ are overestimated erroneously. The fact is that the flare activity of EV Lac increased considerably in the last years in comparison with 1960 [16]; therefore it is natural now to expect the star to have a more powerful 
chromosphere radiating optically thicker and therefore wider lines. Furthermore, Wilson has measured $\mathrm{H} \gamma$ and higher members of Balmer series where self-absorption is less than at $H \alpha$.

\section{Conclusion}

The available data allow us to affirm that the rotational velocities of UV Cet-type flare stars are considerably smaller than those of young OB-stars; probably they do not exceed 25-30 km/sec. Note that according to Krzeminski [1], the 'photometric' rotational velocity of the emission dwarf $\mathrm{BD}+34^{\circ} 106(\mathrm{dM0e})$ is $15 \mathrm{~km} / \mathrm{sec}$ and that of HDE 234677 (dK7e) is $10 \mathrm{~km} / \mathrm{sec}$. If one extrapolates the dependence of mean rotational velocity of main-sequence stars on spectral classes from $\mathrm{G}$ through $\mathrm{K}$ up to M-stars with a smooth curve according to statistics [17] then one would expect a red dwarf rotational velocity to be not higher than a few $\mathrm{km} / \mathrm{sec}$.

\section{Acknowledgements}

I am very grateful to Dr. P. F. Chugainov, Dr. A. A. Bojarchuk, Dr. G. H. Herbig, Dr. R. P. Kraft, Dr. W. Krzeminski, Prof. A. B. Severny and Dr. A. Slettebak for stimulating discussions.

\section{References}

[1] W. Krzeminski: 1969, Low Luminosity Stars (ed. by S. S. Kumar).

[2] P. F. Chugainov: 1966, IBVS N122.

[3] A. D. Andrews: 1966, Publ. Astron. Soc. Pacific 78, 324, 542.

[4] P. F. Chugainov: 1968, Proceedings of the IVth Colloquium on Variable Stars, Budapest.

[5] R. E. Gershberg: 1968, Proceedings of the IVth Colloquium on Variable Stars, Budapest.

[6] E. Schatzman: 1965, Kleine Veröffentlichungen der Remeis-Sternwarte Bamberg, Bd IV, N40, 17.

[7] H. L. Johnson: 1965, Astrophys. J. 141, 170.

[8] T. Tsuji: 1969, Low Luminosity Stars (ed. by S. S. Kumar).

[9] C. S. Higgins, L. H. Solomon, and F. M. Bateson: 1968, Austr. J. Phys. 21, 725.

[10] D. Reuyl: 1943, Astrophys. J. 97, 186.

[11] P. van de Kamp and S. L. Lippincott: 1949, Astron. J. 55, 16.

[12] P. van de Kamp: 1969, Publ. Astron. Soc. Pacific 81, 5.

[13] S. Cristaldi, G. Godoli, M. Narbone, and M. Rodonó: 1968, Proceedings of the IVth Colloquium on Variable Stars, Budapest.

[14] P. F. Chugainov et al:: 1969, IBVS N343.

[15] O. C. Wilson: 1961, Publ. Astron. Soc. Pacific 73, 15.

[16] P. F. Chugainov: 1969, Izv. Crimean Astrophys. Obs. 40.

[17] A. A. Bojarchuk and I. M. Kopylov: 1958, Astron. J. U.S.S.R. 35, 804.

\section{Discussion}

Jordahl: Kunkel (unpublished dissertation, 1967) has done an extensive study of the statistics of flares on three stars: AD Leo, YZ CMi, and Wolf 359, with 25, 25 and 29 flares recorded in total observing times of 90,59 , and 30 hours, respectively. He finds that a Poisson model fits the observations of AD Leo and YZ CMi quite well, where the fit is to the probability that $n$ flares brighter than magnitude $m$ occur per unit time. For Wolf 359 (the faintest of the three objects) the flares tend to be much shorter in time scale and hence difficult to identify unambiguously with the sequential filter- 
sampling technique used. In any case the flare frequency increases rapidly as the detection threshold improves. The concept of a 'mean time between flares' is meaningless unless accompanied by a statement of the detection limit. These statistics are from his study in the V filter of the UBV system, which is much more sensitive to flares than the $B$ or $V$ filters.

These statistics would seem to argue against any interpretation of a 'mean time between flares' in terms of stellar rotation.

Gershberg: Chugainov's statistics which suggest a quasiperi odicity of flares involve 28 flares of UV Cet and 24 flares of YZ CMi (see Non-Periodic Phenomena in Variable Stars (ed. by L. Detre), Budapest, 1969, p. 112 and 127). Therefore the volumes of data in Chugainov's and Kunkel's statistics are similar, but Chugainov has considered stronger flares on the average. He has obtained a periodicity not in the strict sense but the existence of flare groups which are prolonged up to 1-2 hours and separated by a characteristic time interval. If on a rotating star you have one active region where flares occur stochastically you shall have such a time distribution of flares. But if there are several active regions on the star the modulation effect of stellar rotation on the total time distribution of flares can be found with the thin analysis only. This modulation effect can also be smoothed out by the flare detection threshold improvement which allows the registration of flares in weaker active regions and acts as a real increase of active region number on the star. It is known that the flare detection threshold can be improved by using the U-band and by observing absolutely fainter stars. Up to this time Kunkel's extensive observations were not analyzed by the autocorrelation method and therefore they cannot be regarded now as an argument against the interpretation of time distribution of flares in terms of stellar rotation. It should be noted that according to Chugainov the UV Cet flare statistics ( 28 events) can be represented by a Poisson model and quasiperiodicity manner with the same success. 